\title{
How the white tiger lost its color, but kept its stripes
}

\author{
XU Xiao \& LUO Shu-Jin* \\ College of Life Sciences, Peking-Tsinghua Center for Life Sciences, Peking University, Beijing 100871, China
}

Received May 28, 2014; accepted June 9, 2014; published online June 27, 2014

Citation: $\quad$ Xu X, Luo SJ. How the white tiger lost its color, but kept its stripes. Sci China Life Sci, 2014, 57: 1041-1043, doi: 10.1007/s11427-014-4697-z

The fantastic range of animal coloration is produced by pigment particles of colored material or microscopic structures in scales, bristles or feathers that give rise to brilliant iridescent colors. In mammals, except for a few exceptions involving nano- or micro-scale structural color (e.g., the blue faces of male baboons), fur color is determined by the pigment melanin. Melanin is synthesized and stored in melanosomes, a lysosomal organelle of melanocyte, and transferred to adjacent keratinocytes and hairs. There are two types of melanin: eumelanin (black to brown) and pheomelanin (red to yellow). The amount, ratio and distribution of eumelanin and pheomelanin in hair determine the coat color of an animal: everything from bright white to light cream to red to jet black.

The white tiger is a rare color morph of the Bengal tiger (Panthera tigris tigris). Relative to their orange counterparts, white tigers lack pheomelanin, with minor (or no) change in eumelanin, and have white fur interspersed with sepia-brown stripes. This contrast between light and dark gives the white tiger a striking appearance, but has also driven its extinction in the wild due to trophy hunting. Today, all white tigers alive are in captivity and descendants of a single male white tiger (named Mohan) captured in India in 1951.

Given sporadic but consistent observation of white tigers in the wild over centuries of records, it is reasonable to speculate that the white tiger coloration is likely a stable genetic trait in a recessive manner. Data from white tiger captive breeding has confirmed that the striped white coat color is inherited as an autosomal recessive trait [1]. Based

*Corresponding author (email: luo.shujin@pku.edu.cn) on the resemblance of white tigers to the chinchilla variant in mice, Robinson [1] postulated that white tigers arose because of a recessive allele at the chinchilla locus (now known as the tyrosinase gene).

$\mathrm{Xu}$ et al. [2] tested Robinson's hypothesis by sequencing the TYR (tyrosinase) gene along with other key coat-color-associated genes MCIR (melanocortin 1 receptor), ASIP (agouti signaling protein), TYRPI (tyrosinase-related protein 1) and SLC7A11 (solute carrier family 7 , member 11) in both white and orange tigers. No genetic association was detected among these genes, suggesting a different mechanism entirely. Next, a genome-wide association study (GWAS) was conducted in a two-generation family of 16 tigers segregating at the white color trait based on whole genome sequencing and restriction-site-associated DNA sequencing (RAD-seq). The approach mapped the white tiger coat color trait to the gene SLC45A2 (solute carrier family 45, member 2). SLC45A2 encodes a 12transmembrane transporter, whose mutations have been linked to oculocutaneous albinism in people, lowland gorillas and Doberman pinscher dogs, and variable hypopigmentation types in mice (underwhite), horses (cream), chickens (silver) and fish. Further, an alanine to valine substitution (A477V) was identified in SLC45A2 and found to be co-segregated with the white coat color trait. This A 477V mutation in SLC45A2 was validated in an additional 130 unrelated tiger individuals. A477V is located in the evolutionary conserved amino acid residue site, and predicted to affect the function of the SLC45A2 protein by three-dimensional protein structure simulation [2]. $\mathrm{Xu}$ et al. [2] concluded that white tigers are caused by the A477V mutation in the SLC45A2 gene. 


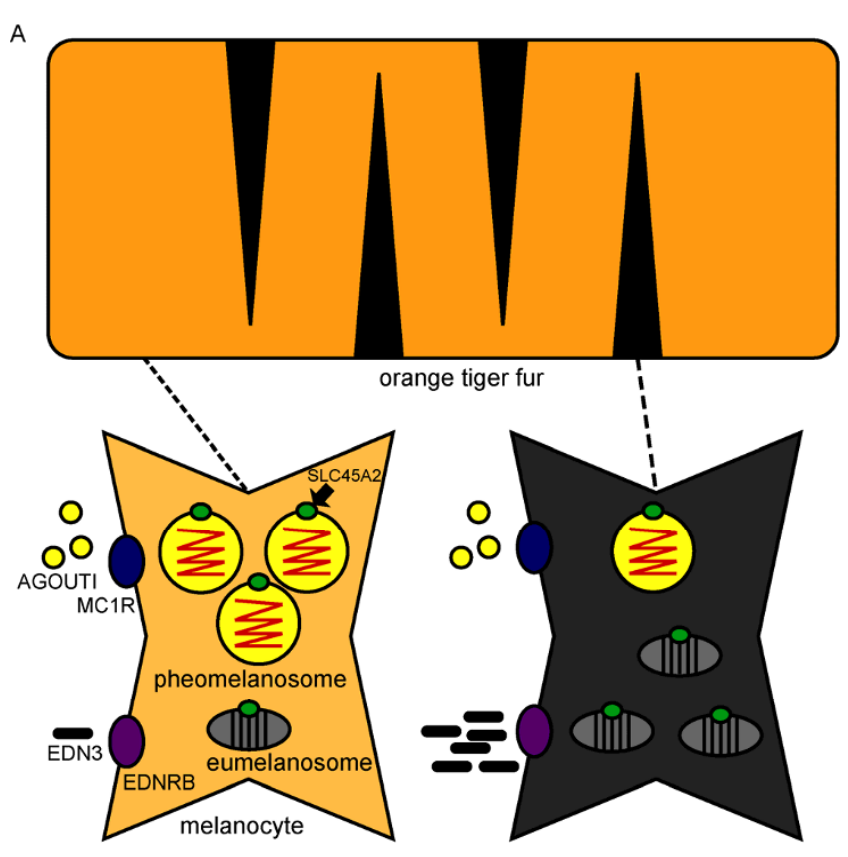

$\mathrm{B}$

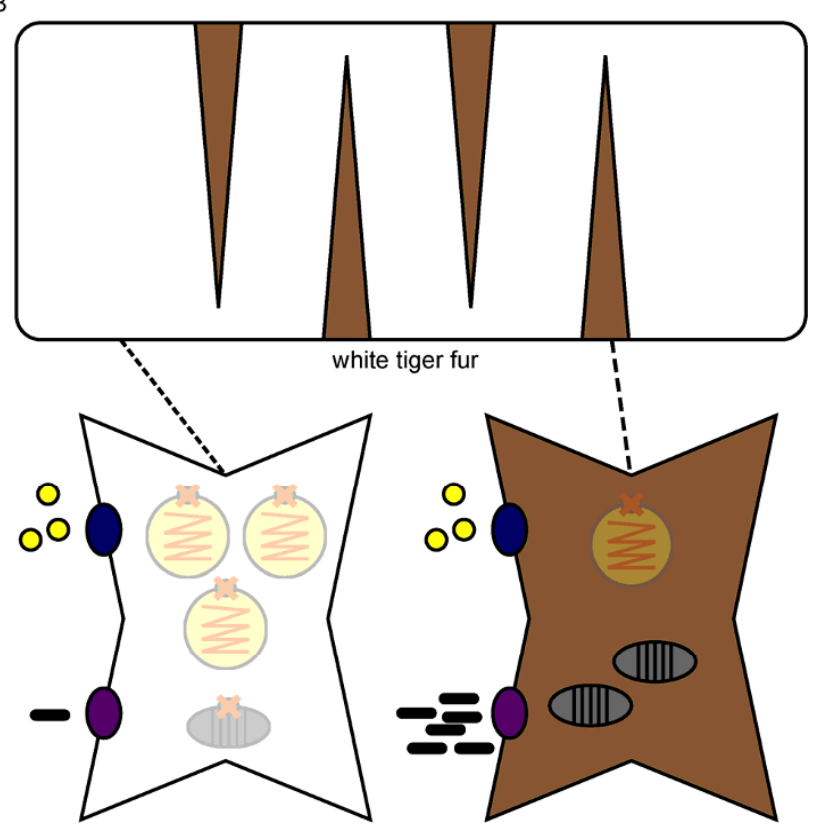

Figure 1 A proposed model for tiger coat color formation through interaction of Mclr and Ednrb signaling pathways under the effect of Slc45a2. A, In an orange tiger, Slc45a2 maintains proper $\mathrm{pH}$ in melanosomes, the $M c 1 r$ pathway engages in downstream eumelanin and pheomelanin syntheses, and Edn3 stimulates eumelanin synthesis through the Ednrb pathway; the high level of Edn3 expression increases eumelanin production and suppresses pheomelanin in striped fur. B, In a white tiger, melanin synthesis is abolished by Slc45a2 deficiency in background fur leading to white coloration, but eumelanin synthesis is sustained by high expression of $E d n 3$ in striped fur.

SLC45A2 is a proton transporter located on the membrane of melanosomes, and regulates organelle $\mathrm{pH}$ homeostasis along with the melansome acidifier V-ATPase [3]. The activity of TYR, a rate-limiting enzyme for melanin biosynthesis, is dependent on proper organelle $\mathrm{pH}$ in the melanosome [4]. Therefore, the way SLC45A2 mediates melanin synthesis is to affect TYR activity by regulating melanosome $\mathrm{pH}[3,4]$. Given that TYR catalyzes lysine into L-DOPA before the melanin synthesis pathway diverges into eumelanin or pheomelanin routes, it is natural to expect that mutations in SLC45A2 would equally affect both eumelanin and pheomelanin, as observed in oculocutaneous albinism in people, lowland gorillas and Doberman pinscher dogs. However, this expectation does not hold in white tigers because pheomelanin is thoroughly inhibited and eumelanin is less (or not) affected (a similar situation has been reported in the cream horse and silver chicken). One plausible explanation is that SLC45A2 might not only transport protons, but also transport cysteine, essential for pheomelanin biosynthesis and irrelevant to the eumelanin pathway. In this scenario, the loss-of-function of SLC45A2 (e.g., gorilla albinism mutation) would block the biosynthesis of both eumelanin and pheomelanin; the moderate defect of SLC45A2, which retains a partial transporting function, may greatly inhibit pheomelanin (both TYR activity and cysteine supply are insufficient), but compromise eumelanin in only a moderate manner. The white tiger mutation (A477V) is predicted to reduce the cavity size of the transportation tunnel, consistent with speculation about its moderate defect on SLC45A2.

Alternatively, the mechanisms of mammalian coat pattern formation may shed light on the pigmentation of white tigers (Figure 1). In a white tiger, dark pigment is restricted to the stripes. Patterned coats are a prominent feature in felids, such as cheetah spots, leopard rosettes and tiger stripes. A two-stage model of coat pattern formation has recently been discovered: a pattern-specifying stage which sets a periodic pre-pattern during early skin development, and a pattern-sustaining mechanism which maintains coat pattern throughout the animal's life [5]. A recent study on felid coat pattern formation has identified such a key gene, $E d n 3$, involved in differential expression and the later pattern-sustaining stage [5]. Edn3 encodes a paracrine hormone belonging to the endothelin family and is mostly expressed by mesenchymal cells. Edn3 plays an essential role in the differentiation and proliferation of melanocytes, and could also stimulate eumelanogenesis via TYR, TYRP1, Dct and other key pigment gene activation [5]. For felid species (domestic cat, leopard cat and cheetah), Edn3 expression is increased in dark-colored regions compared to light-colored regions. The pattern-sustaining mechanism is most likely to be conserved among felids, and perhaps tigers have preserved the region-specific expression pattern of $E d n 3$ in the striped area.

Putting these lines of evidence together, we suggest that the A477V mutation in SLC45A2 may diminish melanin biosynthesis over the entire coat in white tigers, but the high expression of Edn3 sustains eumelanogenesis in some regions according to pattern-specifying mechanisms, resulting 
in white tigers' iconic sepia-brown stripes. These exciting results are the closest we have come to understand the striking coat of a white tiger and how it manages to lose its orange color but retain its stripes.

1 Robinson R. The white tiger of Rewa and gene homology in the Felidae. Genetica, 1969, 40: 198-200

2 Xu X, Dong GX, Hu XS, Miao L, Zhang XL, Zhang DL, Yang HD, Zhang TY, Zou ZT, Zhang TT, Zhuang Y, Bhak J, Cho YS, Dai WT, Jiang TJ, Xie C, Li R, Luo SJ. The genetic basis of white tigers. Curr Biol, 2013, 23: 1031-1035

3 Dooley CM, Schwarz H, Mueller KP, Mongera A, Konantz M,
Neuhauss SC, Nüsslein-Volhard C, Geisler R. Slc45a2 and $\mathrm{V}$-ATPase are regulators of melanosomal $\mathrm{pH}$ homeostasis in zebrafish, providing a mechanism for human pigment evolution and disease. Pigment Cell Melanoma Res, 2013, 26: 205-217

4 Ancans J, Tobin DJ, Hoogduijn MJ, Smit NP, Wakamatsu K, Thody AJ. Melanosomal $\mathrm{pH}$ controls rate of melanogenesis, eumelanin/ phaeomelanin ratio and melanosome maturation in melanocytes and melanoma cells. Exp Cell Res, 2001, 268: 26-35

5 Kaelin CB, Xu X, Hong LZ, David VA, McGowan KA, Schmidt-Küntzel A, Roelke ME, Pino J, Pontius J, Cooper GM, Manuel H, Swanson WF, Marker L, Harper CK, van Dyk A, Yue B, Mullikin JC, Warren WC, Eizirik E, Kos L, O'Brien SJ, Barsh GS, Menotti-Raymond M. Specifying and sustaining pigmentation patterns in domestic and wild cats. Science, 2012, 337: 1536-1541

Open Access This article is distributed under the terms of the Creative Commons Attribution License which permits any use, distribution, and reproduction in any medium, provided the original author(s) and source are credited. 